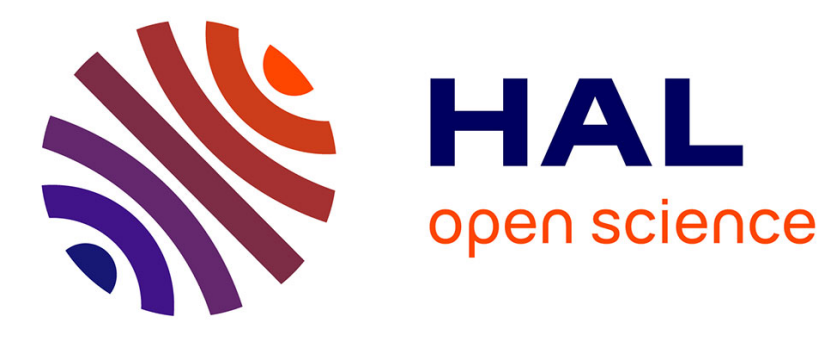

\title{
A new Brewster angle microscope
}

Clarisse Lheveder, Sylvie Henon, Raymond Mercier, Gérard Tissot, Pierre

Fournet, Jacques Meunier

\section{To cite this version:}

Clarisse Lheveder, Sylvie Henon, Raymond Mercier, Gérard Tissot, Pierre Fournet, et al.. A new Brewster angle microscope. Review of Scientific Instruments, 1998, 69 (3), pp.1446-1450. 10.1063/1.1148779. hal-00873392

\section{HAL Id: hal-00873392 \\ https://hal-iogs.archives-ouvertes.fr/hal-00873392}

Submitted on 15 Oct 2013

HAL is a multi-disciplinary open access archive for the deposit and dissemination of scientific research documents, whether they are published or not. The documents may come from teaching and research institutions in France or abroad, or from public or private research centers.
L'archive ouverte pluridisciplinaire HAL, est destinée au dépôt et à la diffusion de documents scientifiques de niveau recherche, publiés ou non, émanant des établissements d'enseignement et de recherche français ou étrangers, des laboratoires publics ou privés. 


\title{
A new Brewster angle microscope
}

\author{
C. Lheveder and S. Hénon a) \\ Laboratoire de Physique Statistique de l'ENS, URA 1306 du CNRS, associé aux Universités Paris VI et VII, \\ 24 rue Lhomond, 75231 Paris, Cedex 05, France \\ R. Mercier, G. Tissot, and P. Fournet \\ Institut d'Optique Théorique et Appliquée, URA 14 du CNRS, Université de Paris-Sud, BP 147, \\ 91403 Orsay Cedex, France \\ J. Meunier \\ Laboratoire de Physique Statistique de l'ENS, URA 1306 du CNRS, associé aux Universités Paris VI et VII, \\ 24 rue Lhomond, 75231 Paris, Cedex 05, France
}

(Received 21 October 1997; accepted for publication 15 December 1997)

\begin{abstract}
We present a new Brewster angle microscope for the study of very thin layers as thin as monolayers, using a custom-made objective. This objective avoids the drawbacks of the models existing at the present time. Its optical axis is perpendicular to the studied layer and consequently gives an image in focus in all the plane contrary to the existing models which give images in focus along a narrow strip. The objective allows one to obtain images with a good resolution (less than $1 \mu \mathrm{m}$ ) without scanning the surface, at the video frequency, allowing for dynamic studies. A large frontal distance associated with a very large aperture is obtained by using a large lens at the entrance of the objective. (C) 1998 American Institute of Physics. [S0034-6748(98)03603-X]
\end{abstract}

\section{INTRODUCTION}

The Brewster angle microscope, introduced six years ago for the study of monolayers at flat liquid interfaces between transparent and isotropic media (such as air and water), ${ }^{1}$ is now a technique that is commonly used in laboratories. It is a noninvasive method for the study of monolayers which do not absorb a significant amount of light, i.e., most of the commonly studied monolayers. The technique avoids the drawbacks of fluorescence microscopy and, in some cases, reveals characteristics of the monolayers that cannot be observed by this last method, such as the lattice anisotropy. ${ }^{2,3}$

Brewster angle microscopy is a microscopy technique based upon the characteristics of the reflection of the light at the Brewster angle. The interface to be studied is illuminated by a parallel laser beam polarized in the plane of incidence $(p)$. The incidence angle is the Brewster angle, which implies that no light is reflected if the interface is perfect, i.e., if there is no interfacial layer and no roughness: the refractive index changes abruptly from the refractive index of one medium to that of the second medium at the level of the interface (Fresnel interface). For a real interface having a transition region in which the refractive index changes smoothly from one value to another, the reflected intensity at the Brewster angle is a minimum but does not vanish completely. It depends strongly on the interfacial properties such as the molecular density and the optical anisotropy in the interfacial layer. In this way, an image of the interface can be formed through a microscope inclined at the Brewster angle,

\footnotetext{
a) Present address: LBHP URA 343 du CNRS, associé aux Université Paris VI et VII, affilié à l'INSERM, 2 place Jussieu, case 7056, 75251 Paris Cedex 05, France.
}

which collects the reflected light and a part of the light scattered by the interface. An analyzer can be introduced in the light path which allows one to analyze the polarization of the reflected and scattered light, i.e., it allows for the analysis of the surface birefringence. Unfortunately, the angle $\theta$ between the normal to the object plane (the surface) and the optical axis of the microscope increases roughly proportionally to the magnification. This leads to an angle $\theta\left(88.9^{\circ}\right.$ for a $40 \times$ magnification) that excludes the possibility of tilting the detector to obtain an image in focus in all the plane. The image is therefore only in focus in a narrow strip. The higher the resolution of the microscope objective (i.e., larger is its numerical aperture), the narrower the strip in focus becomes. For the objective of the first Brewster angle microscope, ${ }^{1}$ we used an objective with a long frontal distance and a large numerical aperture (magnification: 20, numerical aperture: $0.4)$ to obtain a good spatial resolution in the plane of the water $(\sim 1 \mu \mathrm{m})$. In order to obtain a complete image, it was necessary to scan 25 strips that are in focus successively, each strip taking $0.1 \mathrm{~s}$; the entire image is reconstructed by placing these 25 strips side by side. ${ }^{1}$ It thus takes about $2.5 \mathrm{~s}$ to acquire a complete image. This acquisition time can be reduced by reducing the number of strips, i.e., the field of view. The drawback of this technique results from the delay between two strip acquisitions which produces artifacts on images of moving interfaces (Fig. 1) and the delay between two images which only allows the study of very slow dynamics. These drawbacks are avoided in a somewhat simpler apparatus introduced shortly after our microscope. ${ }^{4}$ No scanning is used and the images are obtained directly at the video speed (25-50 images/s). However, as is obvious from the above remarks, the quality of the images is poor: they are in focus only in their middle, on one strip. The width of the strip in focus is increased by reducing the numerical aperture 


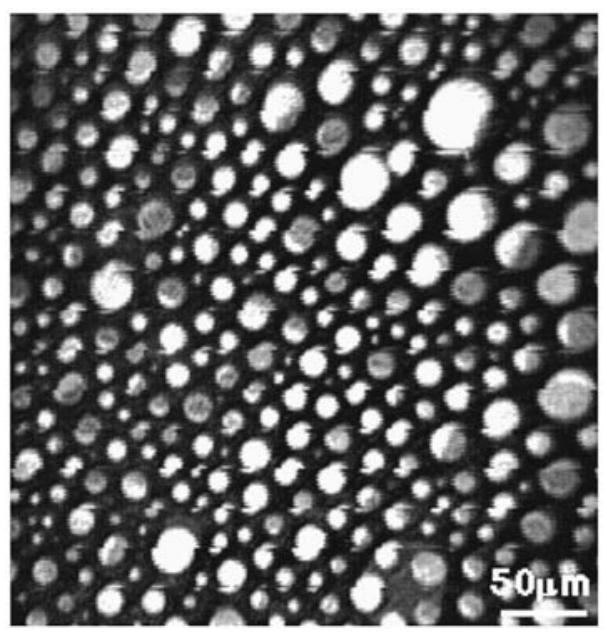

FIG. 1. Example of an image of mobile dense domains (L2 phase in a LE phase) in a monolayer moving quickly. The image is obtained with the scanning Brewster angle microscope (see Ref. 1). The movement of the monolayer introduces a shift on each strip which can be observed on the image.

(and consequently the resolution: the announced resolution, ${ }^{4}$ $1 \mu \mathrm{m}$, is largely overestimated) by using a lens instead of a microscope objective. The Brewster angle microscopes used commonly in laboratories derive from these two first instruments. A simple modification of an ellipsometer by adding a lens as an objective and replacing the detector by a video camera was proposed recently to obtain a low-resolution Brewster angle microscope. ${ }^{5}$ Similarly, an "ellipsometric microscope" was described recently. ${ }^{6}$ These two setups take advantage of the ellipsometric contrast: the information obtained is the ellipticity, i.e., the state of polarization of the light on each pixel of the image. However, these setups have the drawback that obtaining an image takes too much time to take images of fluid films at the free surface of water, for instance, $20 \mathrm{~s}$ with the "ellipsometric microscope." Furthermore, a sharp image of surface is obtained by reducing the optical aperture drastically. This is obtained using a beam expander placed far from the interface. ${ }^{6}$ The announced resolution $(1 \mu \mathrm{m})$ results from a calculation which does not take diffraction into account! The true resolution is probably 10 $\mu \mathrm{m}$ at best, no better than the resolution of some ellipsometers. $^{7}$

The optics of a Brewster angle microscope which avoids all these different drawbacks must, first, collect the reflected light at the Brewster angle and also detect the maximum of the light scattered by small structures in the monolayer under study. Second, it must form with this light an image in focus of the plane of the interface on the sensitive element of a video camera. Third, for technical reasons, a long frontal distance is necessary to study monolayers at the free surface of water.

We propose here a new Brewster angle microscope, which satisfies these three criteria; this is obtained by using a special custom-made objective. This objective has a vertical symmetry axis (it is not tilted) and consequently gives an image of the water surface in a plane parallel to this surface. The large frontal distance associated with a very large aperture to collect the light reflected at the Brewster angle and

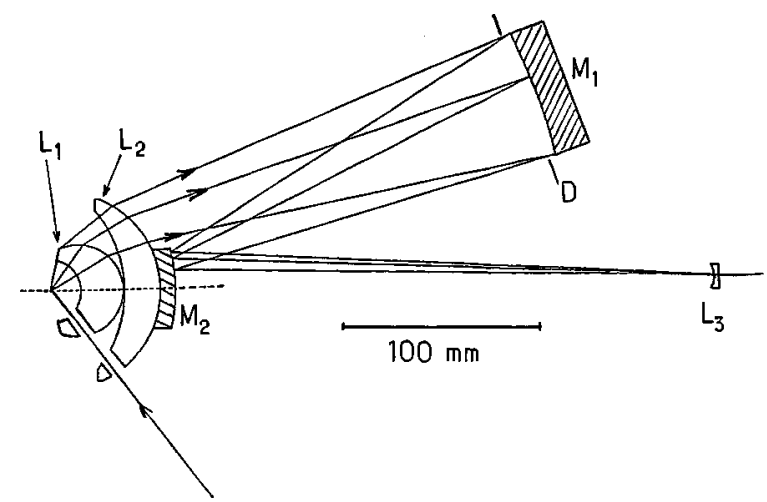

FIG. 2. Schematic drawing of the objective. L1 and L2 are two aplanetic lenses (working at the center of curvature of the first surface and at the Weierstrass points of the second one) which reduce the beam aperture. Mirrors M1 and M2 constitute a Schwarzschild objective with a $\times 6$ magnification. The lens L3 allows to flatten the image field and to adjust the total magnification in the range $20-80$, depending on the position of the lens.

the scattered light are obtained by using a large lens at the entrance of the imaging objective. The very large tilt of the reflected and scattered light beams with respect to the symmetry axis of the objective, at its entrance, is progressively reduced through the objective: at the level of the image, the light beams are almost perpendicular to the image, contrarily to the case of a tilted microscope objective.

Below, we shall describe successively the different parts of the microscope: the objective, the microscope stand, and light source. Then we shall give the optical characteristics of the objective and present images obtained so far with this microscope.

\section{THE OBJECTIVE}

The schematic diagram of the objective is shown Fig. 2. The basic idea of the design is to use an eccentric subaperture of a normal incidence objective, thus keeping the object and image planes perpendicular to the axis of the complete objective. In order to achieve a $1 \mu \mathrm{m}$ resolution at $514.5 \mathrm{~nm}$ (the green wavelength of an argon laser), an aperture for normal incidence of 0.24 is required. Owing to the Brewster angle $\left(53^{\circ}\right)$, the effective useful aperture must be \pm 024 / $\cos \left(53^{\circ}\right)= \pm 0.40$, centered on the Brewster angle. This leads to an aperture angle of $53^{\circ} \pm 23.6^{\circ}$, i.e., a numerical full aperture of 0.97 .

In our objective, this aperture is first reduced by L1 and L2, two aplanetic lenses (working at the centers of curvature of the entrance surfaces and the Weierstrass points of the exit surfaces of both L1 and L2). Each lens magnifies the image and conversely reduces the aperture by its refractive index ( $n=1.628, \mathrm{C} 2036$ flint glass), yielding a 2.65 magnification and a resultant aperture of 0.37 . These two lenses are followed by a Schwarzschild objective ${ }^{8}$ (two concentric spherical mirrors M1 and M2) that gives a further $6 \times$ magnification. Finally, a small diverging lens L3 flattens the image field and allows to adjust the global magnification from 20 to 80, depending upon the position of the lens.

In practice, as can be observed in Fig. 2, only a small part (out of center with respect to the optical axis) of the two first lenses is used to collect both the reflected beam and the 


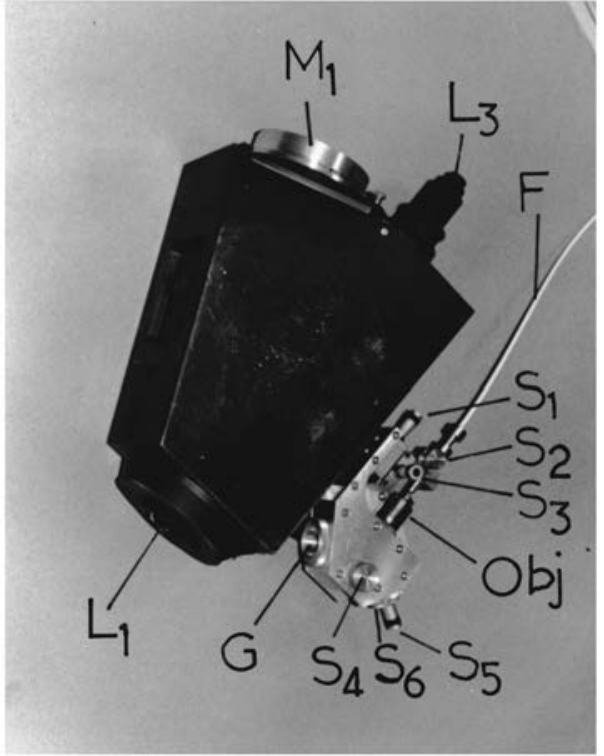

FIG. 3. INVAR mounted structure of the optics of the objective (on the left) and the illumination device (on the right). (A) illumination device: F, single mode optical fiber and the three micrometer screws, S1, S2, S3, allowing its positioning. Obj, microscope objective $G=20$ from newport. S4, screw adjusting the direction of polarization by rotating of the Glan-Taylor polarizer $G$. S5 allows to adjust the angle of incidence of the laser beam without changing its impact point on the monolayer. The screw S6 is for the positioning of a flat mirror. (B) objective: L1, frontal lens. M1, mount of the large spherical mirror M1. L3, last lens of the objective and its mount.

scattered light in a large cone around the reflected beam. However, as the manufacturing and the positioning of nonaxisymmetric lenses is extremely difficult to achieve with the required accuracy, the lenses were made full size and classical centering techniques could thus be used. Mirror M2 was also manufactured full size and was cemented centered onto the lens L2. Mirror M1 was, on the other hand, limited to its useful size: making it larger would have been useless and would have only increased the size and weight of the objective. The mechanical mount of the objective is made of INVAR in order to make it insensitive to room-temperature fluctuations (Fig. 3).

The mirror M1 and the ensemble L2-M2 were first mounted. The extremity of an optical fiber was mechanically centered with respect to L2, on the image side. It served as subresolution source, illuminating backwards the objective, at the working wavelength. Mirror M1 was then adjusted in tilt and position in order to bring the image of the fiber at the center of curvature of the entrance surface of L2 (located using an autocollimator). The theoretical spacing between L1 and L2 had been deduced from the refractive index and the measured thickness and curvature radii of the lenses. This spacing was achieved by an optically polished spacer, placed between the mechanical mounts of the lenses. L1 was then mounted onto the objective and centered as L2 by bringing the image of the fiber at the center of curvature of the entrance surface of L1. The adjustment of the optics was therefore straightforward and could be achieved with great precision.

The pupil is situated on mirror M1, which determines the maximum aperture of the objective. This aperture can be

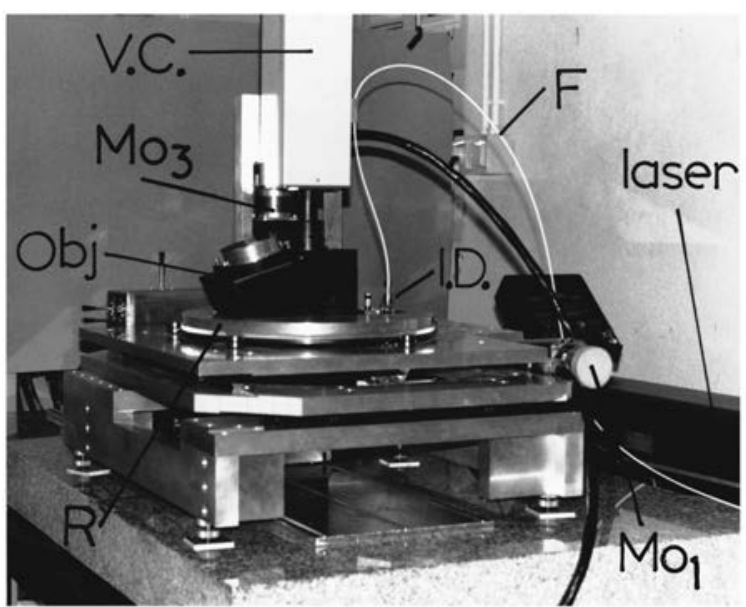

FIG. 4. Picture of the Brewster angle microscope: Obj, objective; I.D., illuminating device; V.C., video camera; $\mathrm{Mo}_{1}$ and $\mathrm{Mo}_{2}$ are step motors translating the objective in the horizontal plane (only $\mathrm{Mo}_{1}$ is visible on the image). $\mathrm{Mo}_{3}$ is a step motor adjusting the focus. A part of the argon laser and of the single mode optical fiber are visible on the right of the image. The objective is fixed on a circular table $(R)$ which can rotate around the optical axis of the objective which is vertical.

reduced by a diaphragm $\mathrm{D}$ placed close to this mirror. Holes were made in the two first lenses for the passage of the Brewster angle illuminating beam.

\section{MICROSCOPE STAND}

The large aperture of the objective requires a high accuracy of positioning and stability of the distance to the objective plane (depth of focus $\sim 1 \mu \mathrm{m}$ ). Focusing is achieved by a step-motor $\left(\mathrm{Mo}_{3}\right.$, Fig. 4). The objective can be displaced in the horizontal plane with two other step-motors $\left(\mathrm{Mo}_{1}\right.$ and $\mathrm{Mo}_{2}$ ) allowing to explore the monolayer or to follow a moving object at the interface. The whole setup can be rotated around the vertical axis of the objective in order to be able to explore the optical anisotropy of the monolayer by rotating the plane of incidence ( $R$, rotating table).

\section{ILLUMINATION DEVICE}

The surface is illuminated with a parallel laser beam, polarized in the plane of incidence. The incidence angle is the Brewster angle. This is achieved using a device fixed on the objective, as shown in Fig. 3. A laser beam is guided from an argon laser (Innova 90 from Coherent) to this device through a single mode optical glass fiber (F). A standard microscope objective $(\mathrm{Obj})$ situated after the fiber gives a parallel beam which is polarized when passed through a Glan-Taylor polarizer $(\mathrm{G})$. Between the microscope objective and the Glan-Taylor, the beam is folded by reflection on a plane mirror in order to reduce the horizontal extension of the device. The end of the glass fiber is positioned at the entry of the microscope objective by three micrometer screws (S1, S2, S3). A fourth one serves to adjust the polarization of the incident light (S4), a fifth one allows us to adjust the angle of incidence of the laser beam without changing its impact point on the monolayer (S5). Finally, a last screw supports the planar mirror and allows for its positioning (S6). 


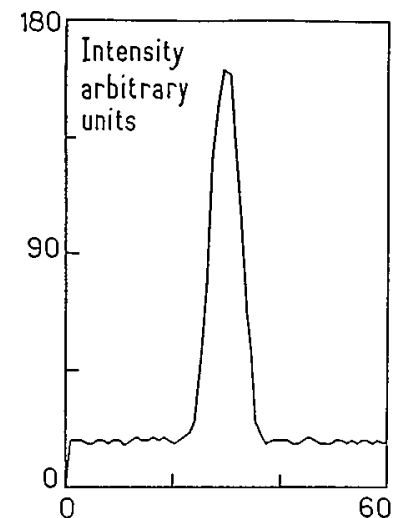

(a)

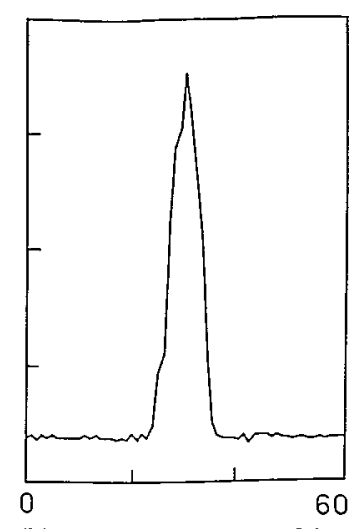

(b)
PIXELS
FIG. 5. Intensity along a straight line (a) perpendicular or (b) parallel to the plane of incidence, vs the position (in pixels on the video camera), for the image of albuminous pin-point (the straight lines pass through the center of the image of the pin-point). The width at half height is 6 pixels, corresponding to a resolution $\sim 0.8$ and $\sim 1 \mu \mathrm{m}$, respectively.

\section{OPTICAL CHARACTERISTICS OF THE OBJECTIVE}

The objective was first tested without the lens L3 (magnification $15.9 \times$ ) and then with lens L3 to obtain different magnifications in the range $20 \times-60 \times$. For these tests, we used a CCD camera $(\mathrm{COHU})$ in order to avoid distorting the

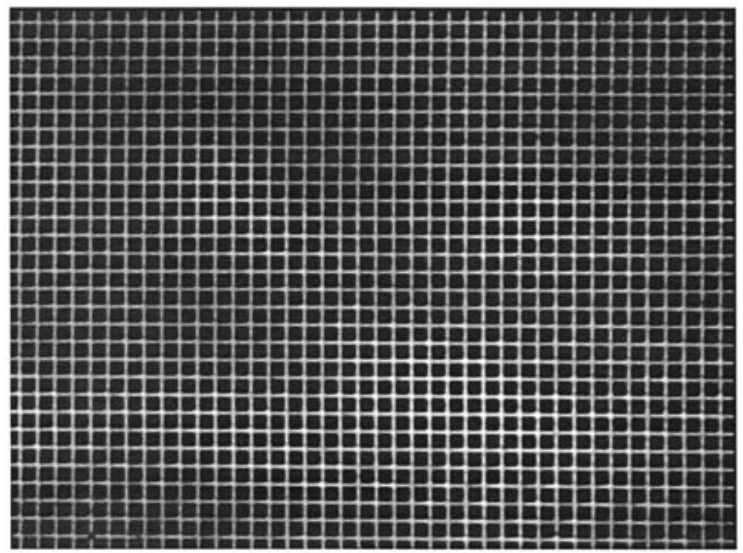

(a)

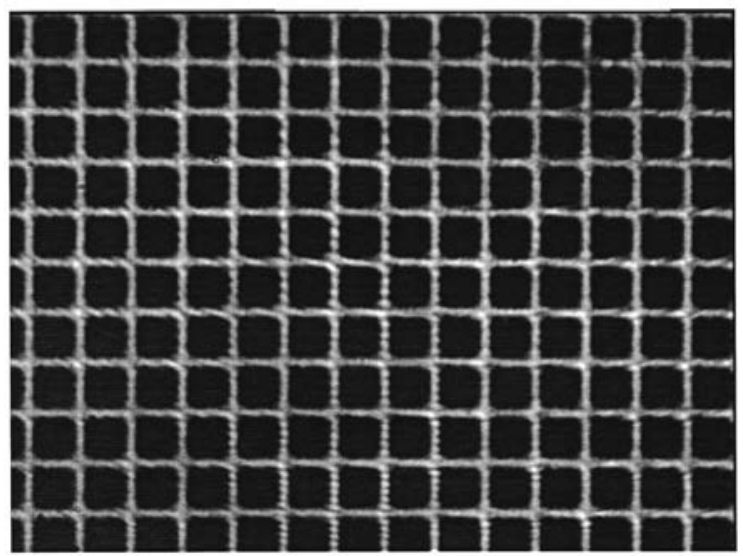

(b)

FIG. 6. Images of a square grid $(10 \mu \mathrm{m} \times 10 \mu \mathrm{m})$ with two different objective magnifications showing a very small pincushion distortion: (a) magnification $\times 16$, (b) magnification $\times 40$.

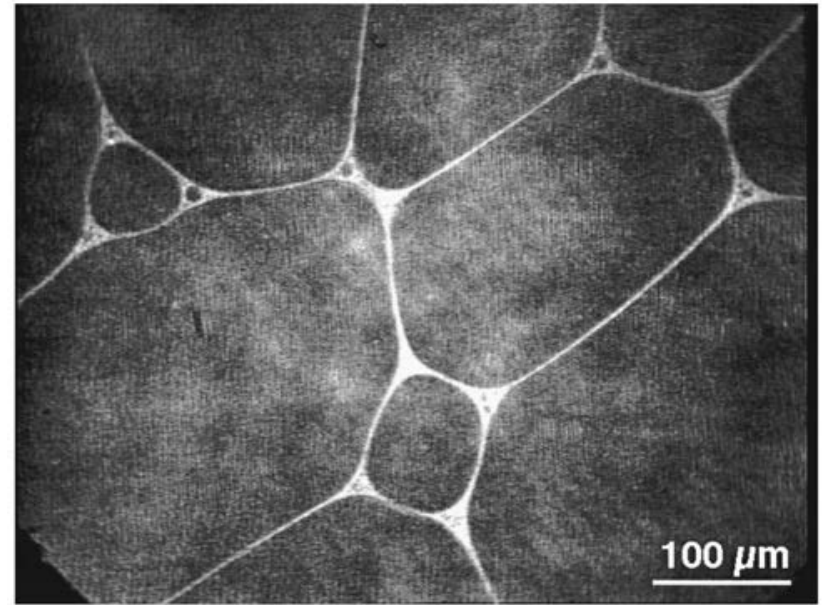

(a)

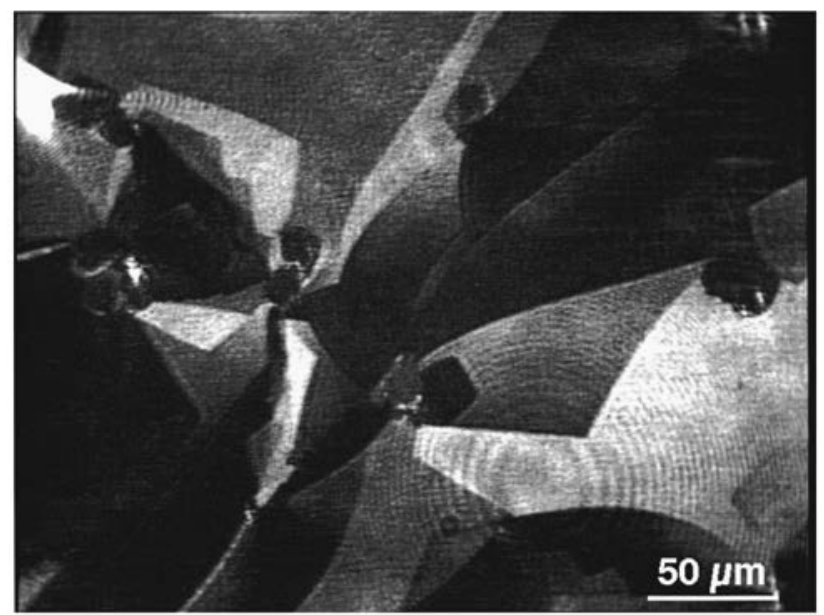

(b)

FIG. 7. (a) Image of a two-dimensional foam obtained at the coexistence between the gas phase and the liquid expanded (LE or L1) phase, in a Langmuir monolayer of myristic acid (room temperature, $p \mathrm{H}=2$, objective magnification $\times 20$ ). (b) Image of the optically anisotropic L2 phase in a monolayer of hexadecanoic acid (room temperature, $p \mathrm{H}=2$, objective magnification $\times 40$ ), obtained by adding an analyzer in the microscope objective. Large zones with a constant tilt azimuthal angle appear uniform and are separated by defect lines across which the tilt azimuthal angle varies abruptly.

images. However, due to the video frame grabber we use (NEOTECH IG 24PCI) the magnification is not the same in both directions (it is about $20 \%$ larger in the direction of the video lines than in the vertical direction).

First, the resolution was estimated from the diffraction image at magnification 60 of a luminous pin-point object. This luminous pin-point object is the transmitted light of a laser beam through a hole having a diameter smaller than the wavelength of the light. For this, we used a small hole in the $\mathrm{Al}$ coating of a glass plate. At the maximum objective aperture, the diffraction spot appears circular and its measured diameter at half height is 6 pixels. Taking into account the difference of magnification in the two directions, this corresponds to a resolution of $1 \mu \mathrm{m}$ in the direction of the tilt and $0.8 \mu \mathrm{m}$ in the perpendicular direction [Figs. 5(a) and 5(b)].

As mentioned previously, the objective aperture can be reduced by placing a diaphragm in front of the large mirror of the Schwarzschild. This diaphragm reduces the resolution 
but at the same time it increases the depth of focus and reduces the spurious light diffracted, for instance, by the bottom of the trough containing the liquid.

The distortion of the images formed by the objective was tested by forming the images of a square grid $(10 \mu \mathrm{m} \times 10$ $\mu \mathrm{m}$ mesh) at different objective magnifications [Fig. 6(a): magnification: $\times 16$, Fig. 6(b): magnification: $\times 40]$. Although very difficult to detect, a very small pincushion distortion was observed (the theoretical value is $2 \%$ for the full object field of $0.5 \mathrm{~mm}$ in diameter at $40 \times$ magnification).

As an example of the image one can obtain on a Langmuir monolayer, an image of a two-dimensional foam of a liquid expanded phase (LE or L1) in a gas phase, obtained with this objective, is shown Fig. 7(a) (a monolayer of myristic acid at the free surface of water at $p \mathrm{H}=2$ and at room temperature, objective magnification $\times 20$ ).

In spite of the large thickness of glass crossed by the light (due to the large thicknesses of the two first lenses) and of the large incidence angles at the glass/air surfaces, the objective does not significantly depolarize the light. The addition of an analyzer just before the lens L3 allows to observe the $s$ component of the light reflected by an optically anisotropic monolayer. As an example, Fig. 7(b) shows an image of the anisotropic dense phase L2 of a monolayer of the hexadecanoic acid (room temperature, $p \mathrm{H}=2$, objective magnification: $\times 40$ ), obtained with an analyzer. The optical anisotropy is due to the tilt of the molecules at the air/water interface. The tilt azimuthal angle of the molecules in the monolayer is constant on large zones, which appear almost uniform, and are separated by defect lines through which the tilt azimuthal angle changes abruptly.

\section{DISCUSSION}

This new objective gives an image in focus in the whole plane of the surface under study, with a good spatial resolution $(0.8-1 \mu \mathrm{m}$ according to the direction) and low distortion. It allows one to obtain images of monomolecular plane layers at the video frequency. Its long frontal distance $(\sim 3$ $\mathrm{mm}$ ) allows easily for the study of monolayers at liquid surface. Its optical quality allows for the study of the optical anisotropy of monolayers.

\section{ACKNOWLEDGMENTS}

J. Meunier and S. Hénon thank the "Direction des Recherches Etudes et Techniques" for a financial support (Commande sur Travaux number 92/1448/A000/DRET/DS/ SR) and the CEE for a contract (CHRX-CT 930128). The authors are indebted to G. Colas and J.-C. Maneville for the technical realization of lenses and mirrors, to Y. Sorin for his technical assistance for the mechanics.

${ }^{1}$ S. Hénon and J. Meunier, Rev. Sci. Instrum. 62, 936 (1991).

${ }^{2}$ G. A. Overbeck, D. Hönig, L. Wolthaus, M. Gnad, and D. Möbius, Thin Solid Films 242, 26 (1994).

${ }^{3}$ S. Rivière, S. Hénon, J. Meunier, D. K. Schwartz, M.-W. Tsao, and C. Knobler, J. Chem. Phys. 101, 10045 (1994).

${ }^{4}$ D. Hönig and D. Möbius, J. Phys. Chem. 95, 4590 (1991).

${ }^{5}$ R. Reiter, H. Motschmann, H. Orendi, A. Nemetzand, and W. Knoll, Langmuir 8, 1784 (1992).

${ }^{6}$ H. K. Pak and B. M. Law, Rev. Sci. Instrum. 66, 4972 (1995); B. M. Law and H. K. Pak, J. Opt. Soc. Am. A 13, 379 (1996).

${ }^{7}$ M. Erman and J. B. Theeten, J. Appl. Phys. 60, 859 (1986).

${ }^{8}$ C. R. Burch, Proc. Phys. Soc. 59, 41 (1947); 59, 47 (1947). 